\author{
Article \\ Doi 10.5943/sif/1/1/7 \\ Copyright (c) Mushroom Research Foundation 2016
}

\title{
Lopadostoma fagi (Lopadostomataceae) on Fagus sylvatica from Italy
}

\author{
Daranagama DA ${ }^{1,2,3}$, Camporesi $\mathbf{E}^{5,6,7}$, Liu XZ1, Chamyuang $\mathrm{S}^{2,3}$, Stadler $\mathrm{M}^{4}$, \\ Jingzu $S^{1,2,3}$ and Hyde $K^{2,3}$
}

\author{
1.State Key Laboratory of Mycology, Institute of Microbiology, Chinese Academy of Sciences, No $31^{\text {st }}$ West Beichen \\ Road, Chaoyang District, Beijing, 100101, People's Republic of China. \\ 2.Center of Excellence in Fungal Research, Mae Fah Luang University, Chiang Rai, 57100, Thailand \\ 3.School of Science, Mae Fah Luang University, Chiang Rai, 57100, Thailand. \\ 4.Helmholtz-Zentrum für Infektionsforschung GmbH, Dept. Microbial Drugs, Inhoffenstrasse 7, 38124, Braunschweig, \\ Germany. \\ 5.A.M.B. Gruppo Micologico Forlivese “Antonio Cicognani”, Via Roma 18, Forlì, Italy. \\ 6.A.M.B. Circolo Micologico “Giovanni Carini”, C.P. 314, Brescia, Italy. \\ 7.Società per gli Studi Naturalistici della Romagna,C.P. 144, Bagnacavallo (RA), Italy.
}

Daranagama DA, Camporesi E, Liu XZ, Chamyuang S, Stadler M, Jingzu S, Hyde KD 2016 Lopadostoma fagi (Lopadostomataceae) on Fagus sylvatica from Italy. Studies in Fungi 1(1), 8089, Doi 10.5943/sif/1/1/7

\begin{abstract}
A new record of Lopadostoma fagi is described in Italy from Fagus sylvatica based on morphological and molecular data. It has effused-pulvinate stromata immersed in the host tissue, with a narrow, black, carbonized encasement. The ectostromatic disc is visible as a clypeus and surrounded by the reddish brown bark surface. In the combined phylogenetic analysis of ITS, LSU andRPB2 sequence data, the strain derived from the specimen clustered with other L. fagi strains with high bootstrap support. A morphological description with detailed photographs for L. fagi is provided in this study.
\end{abstract}

Key words - Ascomycetes - phylogeny - Sordariomycetes - taxonomy - Xylariales

\section{Introduction}

Lopadostoma (Nitschke) Traverso (Anthostoma subgen. Lopadostoma Nitschke) was accepted in the Xylariaceae by Læssøe (1994). However the libertella-like asexual morphs of Lopadostoma are more or less similar to those of Diatrypaceae with scolecosporus conidia produced on sympodially proliferating conidiogenesis cells (Ju et al. 1993). Rappaz (1992, 1993) placed Anthostoma decipiens (DC.) Nitschke in Diatrypaceae, which was accepted by Lu and Hyde (2000). The unclear distinction between Xylariaceae and Diatrypaceae has been addressed by several authors (Rogers 1994, Stadler et al. 2013). Recent molecular data have resulted in several changes to the placement of Lopadostoma and Creosphaeria Theiss., which formed a monophyletic clade between Xylariaceae and Diatrypaceae (Jaklitsch et al. 2014, Daranagama et al. 2015, Maharachchikumbura et al. 2015). Thus Senanayake et al. (2015) introduced a new family Lopadostomataceae, in the order Xylariales, to accommodate Lopadostoma and Creosphaeria, based on the phylogeny and also the morphology of the libertella-like asexual morphs of these two genera. 
The morphology of Lopadostoma has been comprehensively studied in Jaklitsch et al. (2014) who accepted 12 species. The sexual morph of Lopadostoma species usually possess pustulateeffuse stromata, immersed in the host which later become erumpent through the bark and appear as blackened areas containing several perithecia, which are clustered in a valsoid arrangement with convergent ostiolar necks. Asci are usually unitunicate, cylindrical, with J+ apical apparatus, with eight uniseriate, dark coloured ascospores, with a straight germ slit (Jaklitsch et al. 2104). The asexual morph of Lopadostoma as reported in Ju et al. (1993) and Jaklitsch et al. (2014) as libertella-like with falcate, unicellular, hyaline conidia.

In this study we examine the phylogenetic placement of our fresh collection with all the known species of Lopadostoma, as well as genera like Anthostomella and Creosphaeria with similar morphology. We have performed a multigene analysis using ITS, LSU and RPB2 sequence data to determine the phylogenetic placement of this species, in which we identified our collection as Lopadostoma fagi Jaklitsch, J. Fourn. \& Voglmayr. A detailed morphological description is provided for the species.

\section{Materials and Method}

Sampling and morphology

A fresh specimen was obtained from Italy on Fagus sylvatica L. Morphological examination and microphotography was carried out as described by Daranagama et al. (2015a, b). Axenic cultures were developed and maintained in Oat Agar (OA) medium for observation and DNA extraction as described by Daranagama et al. (2015a, b).

DNA extraction, PCR and sequencing

DNA was extracted and the ITS, LSU and RPB2 genes were amplified following the protocols outlined by Daranagama et al. (2015). PCR products were visualized in 1\% agarose gel electrophoresis, stained with Goldview (Geneshun Biotech, China) with D2000 DNA ladder (Realtimes Biotech, Beijing, China). All the PCR products were purified according to the company protocols and DNA sequencing was performed using the same primers in an Applied Biosystem 3730 DNA analyzer at Sinogenomax Company, Beijing, China.

Sequence alignment and phylogenetic analyses

Raw sequences were assembled with Contig Express 2003 (Invitrogen, Carlsbad, CA). The assembled consensus sequences were initially aligned with ClustalW and optimized with MAFFT v. 7 using default settings (Katoh \& Standley 2013) (http://mafft.cbrc.jp/alignment/server/) and adjusted manually where necessary. The initial identities of the newly generated sequences were determined by analyzing them with all available type-derived and authentic sequences of Lopadostoma (Jaklitsch et al. 2014). The familial placement was determined by analyzing with strains belong to Lopadostomataceae, Diatrypaceae and Xylariaceae (Table 1).

Evolutionary models for phylogenetic analyses were selected independently for each locus using MrModeltest 2.3 (Nylander 2004) under the Akaike Information Criterion (AIC). ML phylogenetic trees were constructed using RAxML 7.4.2 Black Box (Stamatakis 2006, Stamatakis et al. 2008) available in the CIPRES Science Gateway platform (Miller et al. 2010). For the combined dataset all free modal parameters were obtained using RAxML with ML estimate of 25 per site rate categories. The RAxML software accommodated the GTR model of nucleotide substitution with the additional options of modeling rate heterogeneity $(\Gamma)$ and proportion invariable sites (I).

Phylogenetic trees and data files were viewed in MEGA 5 (Tamura et al. 2011) and Fig tree v1.4 (Rambaut and Drummond 2008). Maximum Likelihood values (equal to or above 50) are indicated above or below nodes. All the sequences generated in this study were deposited in GenBank (Table 1). Faces of fungi numbers are as explained in Jayasiri et al. (2015). 
Table 1 GenBank accession numbers and their strain numbers.

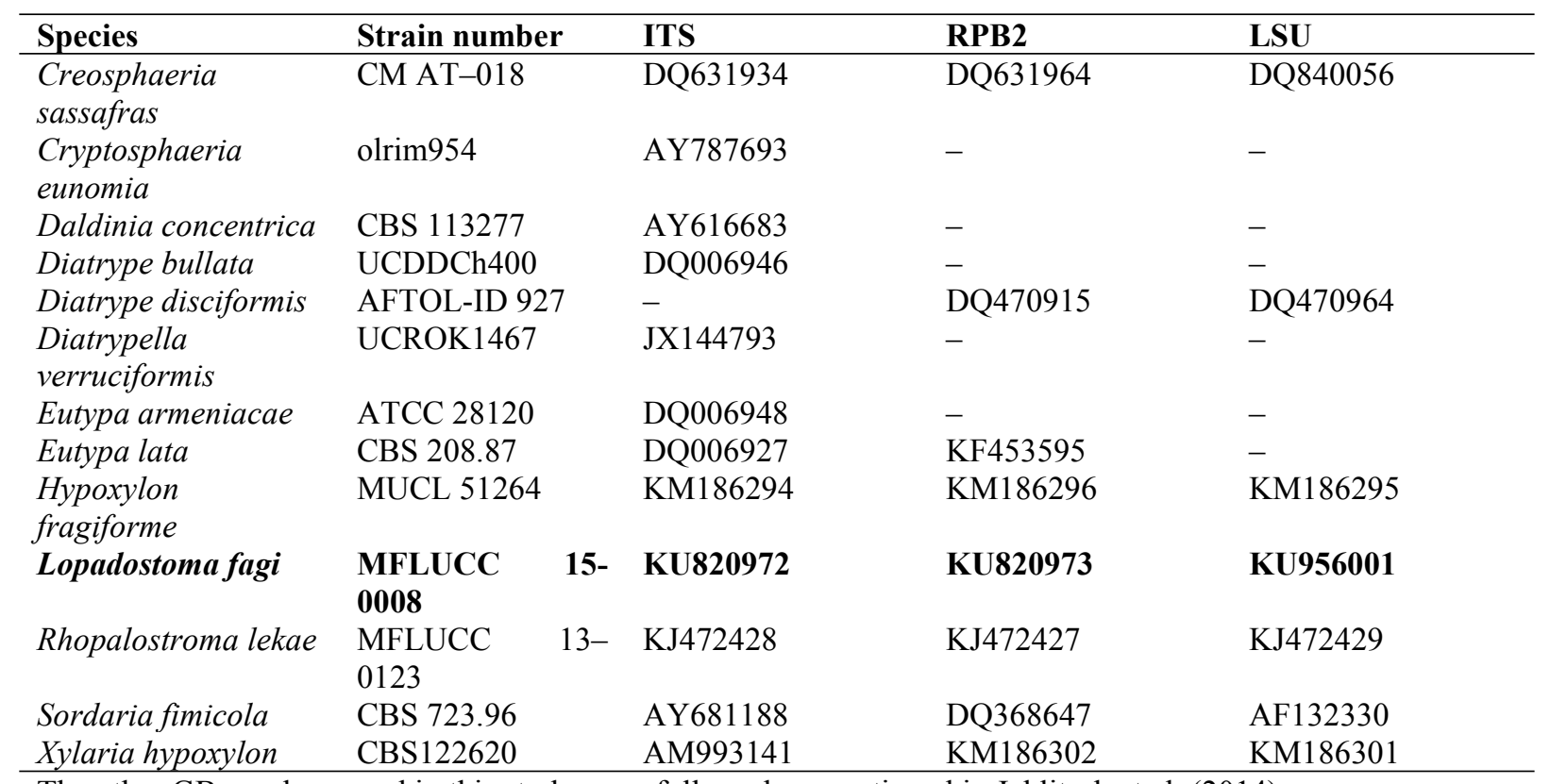

The other GB numbers used in this study were followed as mentioned in Jaklitsch et al. (2014).

\section{Results}

\section{Molecular Phylogenetic Analysis}

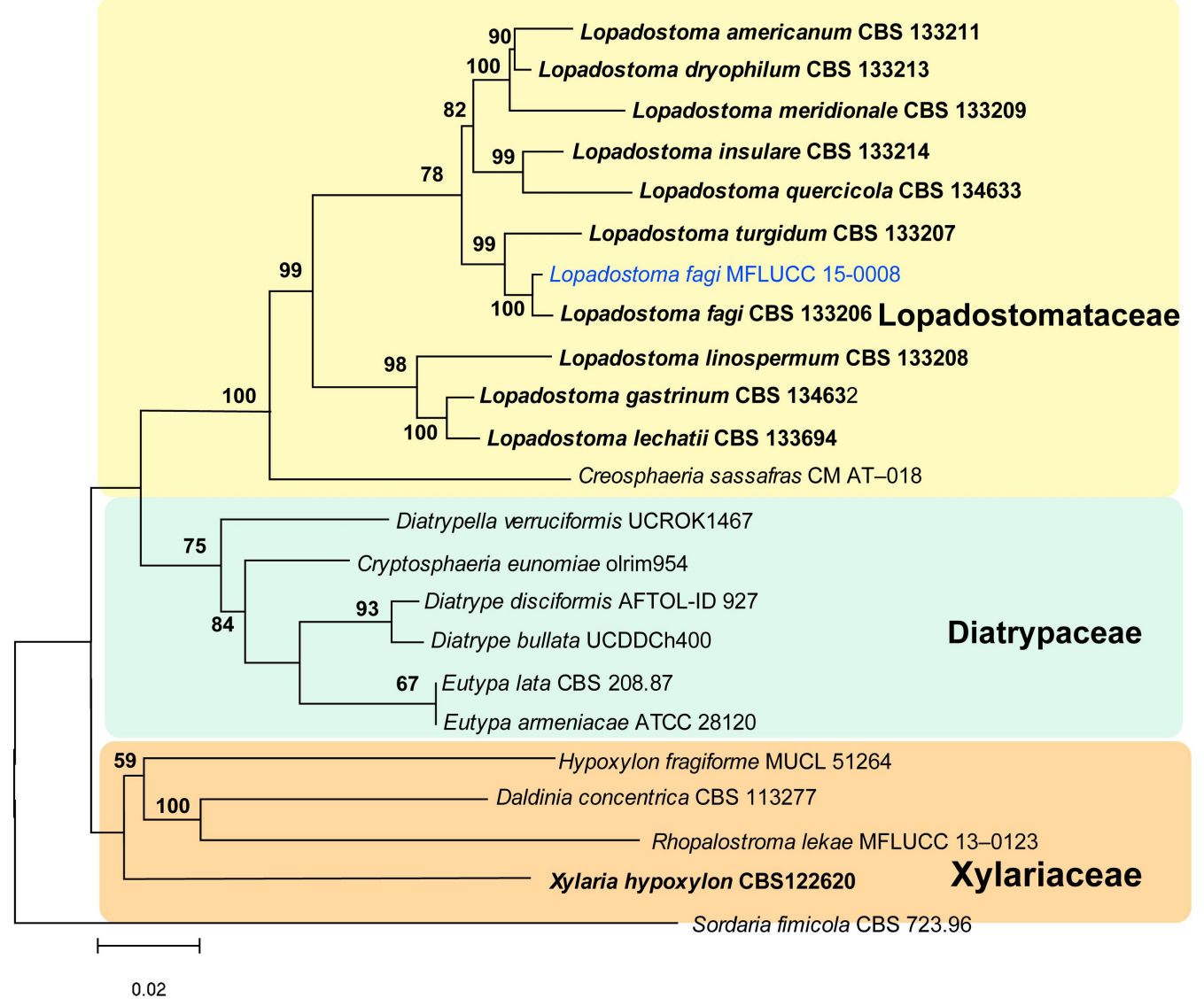

Fig.1. Phylogram inferred from likelihood analysis using combined ITS/LSU/RPB2 sequence data. Strain/culture numbers are given following the taxon names. The new sequences generated in this study are in blue. Strain/culture designations are given following the taxon names. Holo-, neo- or epitype strains/specimens are formatted in bold. The bootstrap support values from likelihood analysis $>50 \%$ from 1000 RAxML replicates are shown above or below the branches. The tree is rooted with Sordaria fimicola. 


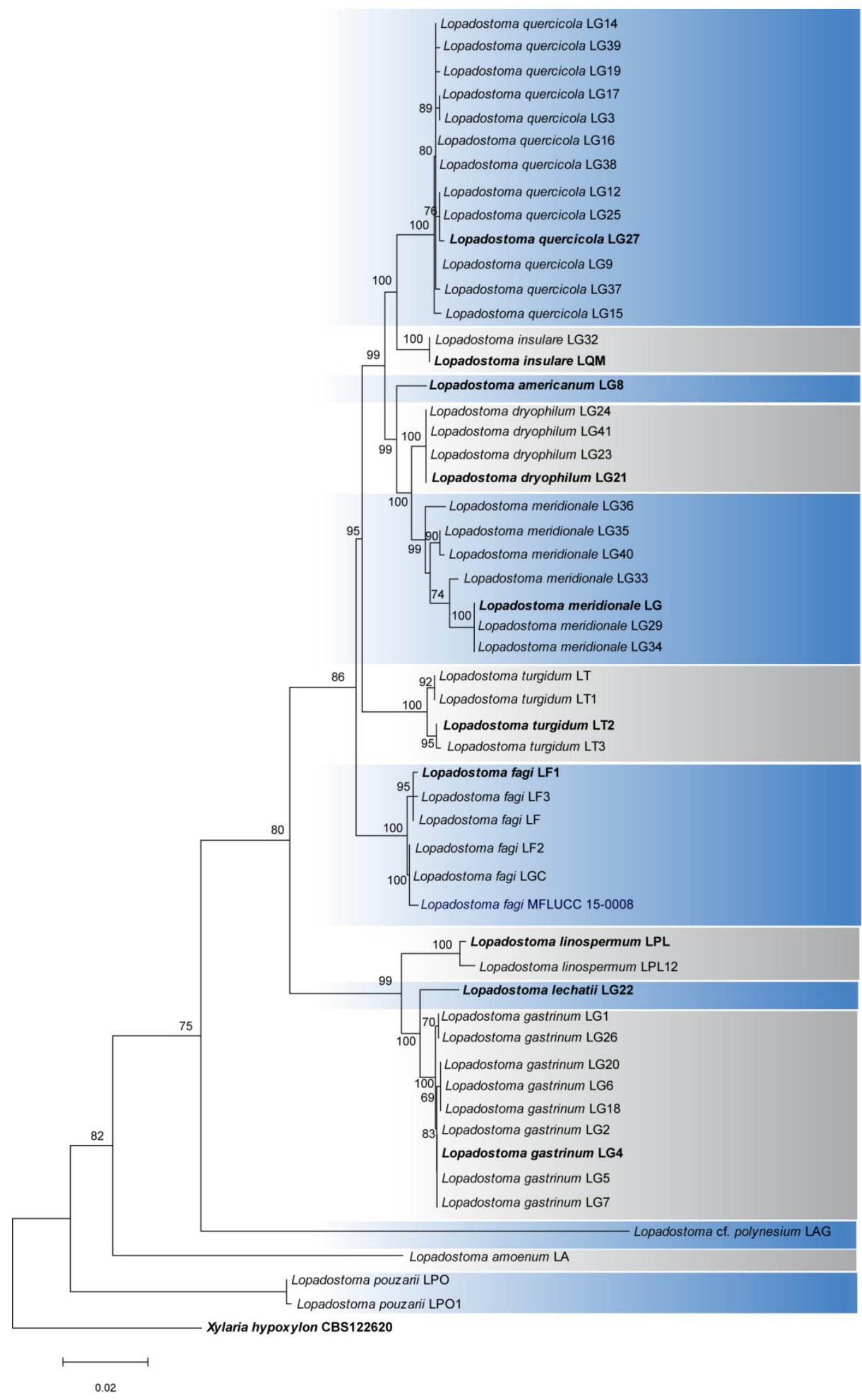

Fig.2. Phylogram inferred from Maximum likelihood analysis using combined ITS-LSU-RPB2 sequence data of Lopadostoma species. Strain/culture numbers are given following the taxon names. The new sequences generated in this study are in blue. Strain/culture designations are given following the taxon names. Holo-, neo- or epitype strains/specimens are formatted in bold. The bootstrap support values from likelihood analysis $>50 \%$ from 1000 RAxML replicates are shown above or below the branches. The tree is rooted with Xylaria hypoxylon. 
The combined alignment of ITS, LSU and RPB2consisted of 2507 total characters with 23 isolates including the out group taxon. All characters were assessed to be unordered and equally weighed. Gaps were treated as missing data. Resolutions of the terminal clades in the multi-locus tree are better than any individual dataset (data not presented). The topology of the resulted ML tree inferred from RAxML is presented as Fig. 1. The phylogenetic data presented in Fig. 1 resolve three monophyletic groups which indicate separate families; Diatrypaceae, Lopadostomataceae and Xylariaceae. Our strain is clearly unrelated to the Xylariaceae or Diatrypaceae families. The latter forms a monophyletic clade as a sister group to Lopadostomataceae. However, in the ML analysis (Fig. 1) this relationship did not obtain branch support above $50 \%$.

In this analysis Lopadostoma forms a monophyletic clade with high bootstrap support. Creosphaeria sassafras is placed singly on a branch sister (100\%) to Lopadostoma. Our strain with high bootstrap support, clustered within Lopadostomataceae (Fig. 1). The ML phylogenetic tree for the genus Lopadostoma was reconstructed using combined ITS-LSU-RPB2 sequence data and the resulted tree is presented as Fig. 2. Molecular data suggests that our strain is related to the $L$. fagi strain from previous studies with high bootstrap support (Fig. 2), which has similar morphological characters. Therefore we have identified this species as Lopadostoma fagi.

\section{Taxonomy}

Lopadostoma fagi Jaklitsch, J. Fourn. \& Voglmayr, in Jaklitsch et al. Persoonia, Mol. Phyl. Evol. Fungi 32: 63 (2014)

Facesoffungi number: FoF 01897

Fig. 3, 4

Saprobic on dead twigs and branches of Fagus sylvatica L. Sexual morph: Stromata 700 $1500 \times 300-800 \mu \mathrm{m}(\bar{x}=1200 \times 500 \mu \mathrm{m}, \mathrm{n}=30)$, effused-pulvinate, immersed in the host and erumpent from bark, bluntly conical, surrounded by a narrow, black, carbonized encasement, appearing as a black line, ectostromatic disc visible as a clypeus and surrounded by reddish brown bark surface, convex, raised, dark grey, entostroma dark, usually black, multi-peritheciate. Ostiole papillate with inconspicuous ostiolar openings. Perithecia $200-800 \times 150-680 \mu \mathrm{m}(\bar{x}=400 \times 520$ $\mu \mathrm{m}, \mathrm{n}=30), 2-5$ clustered in valsoid groups, mono-distichous, subglobose to flask-shaped, at the periphery inclined toward the center, tissue between perithecia loosely arranged, composed of white fungal tissue, mixed with light coloured bark cells, tissue beneath the perithecia compact, black, with short convergent ostiolar necks. Paraphyses are numerous, long, apically free, 1-3.5 $\mu \mathrm{m}$ wide $(\bar{x}=3 \mu \mathrm{m}, \mathrm{n}=30)$, rarely branched. Asci $79.8-103.5 \times 4.6-6.1 \mu \mathrm{m}(\bar{x}=91 \times 5.3 \mu \mathrm{m}, \mathrm{n}=$ 30), unitunicate, 8-spored, cylindrical, pedicellate, ellipsoidal-discoid, inconspicuous apical apparatus typically $2-3.5 \mu \mathrm{m}$ wide. Ascospores $7.5-8.7 \times 3.2-4.2 \mu \mathrm{m}(\bar{x}=7.9 \times 3.9 \mu \mathrm{m}, \mathrm{n}=30)$, uniseriate, oblong to narrowly ellipsoid, symmetrical to slightly inequilateral, unicellular, lacking a dwarf cell, at first hyaline, turning pale brown and dark brown at maturity, smooth-walled, with a straight germ slit across the entire spore length present, when immature with 2 large guttules.

Culture characteristics - Colonies on Difco OA very slow growing, reaching the edge of 9 cm Petri-dishes in 3-3 $1 / 2$ months at $25-27^{\circ} \mathrm{C}$, at first whitish, velvety, azonate, with slightly lobed margins, developing sporulating regions as light yellow mycelial masses in culture; later, reverse turning light orange.

Specimen examined - Italy, Province of Forlì-Cesena, Campigna - Santa Sofia, dead branch of Fagus sylvatica L (Fagaceae), 19 September 2014, E. Camporesi, (MFLU 15-2600, KUN), living culture (MFLUCC 15-0008, KIBCC).

Notes - Lopadostoma fagi is reported as a common species in corticated branches of Fagus sylvatica from Europe (Jaklitsch et al. 2014). This specimen was collected from Italy on the same host. According to the morphological description of L. fagi provided in Jaklitsch et al. (2014) it is characterized in having distinctly papillate ostioles and smaller, narrower ascospores with a straight, full length germ slit. Similar morphological traits were observed in our collection. However, we observed 2-5 perithecia, in stroma more or less in a valsoid configuration and rarely distichous, even though according to Jaklitsch et al. (2014) it is mentioned as (3-) 6-8 in a cluster 

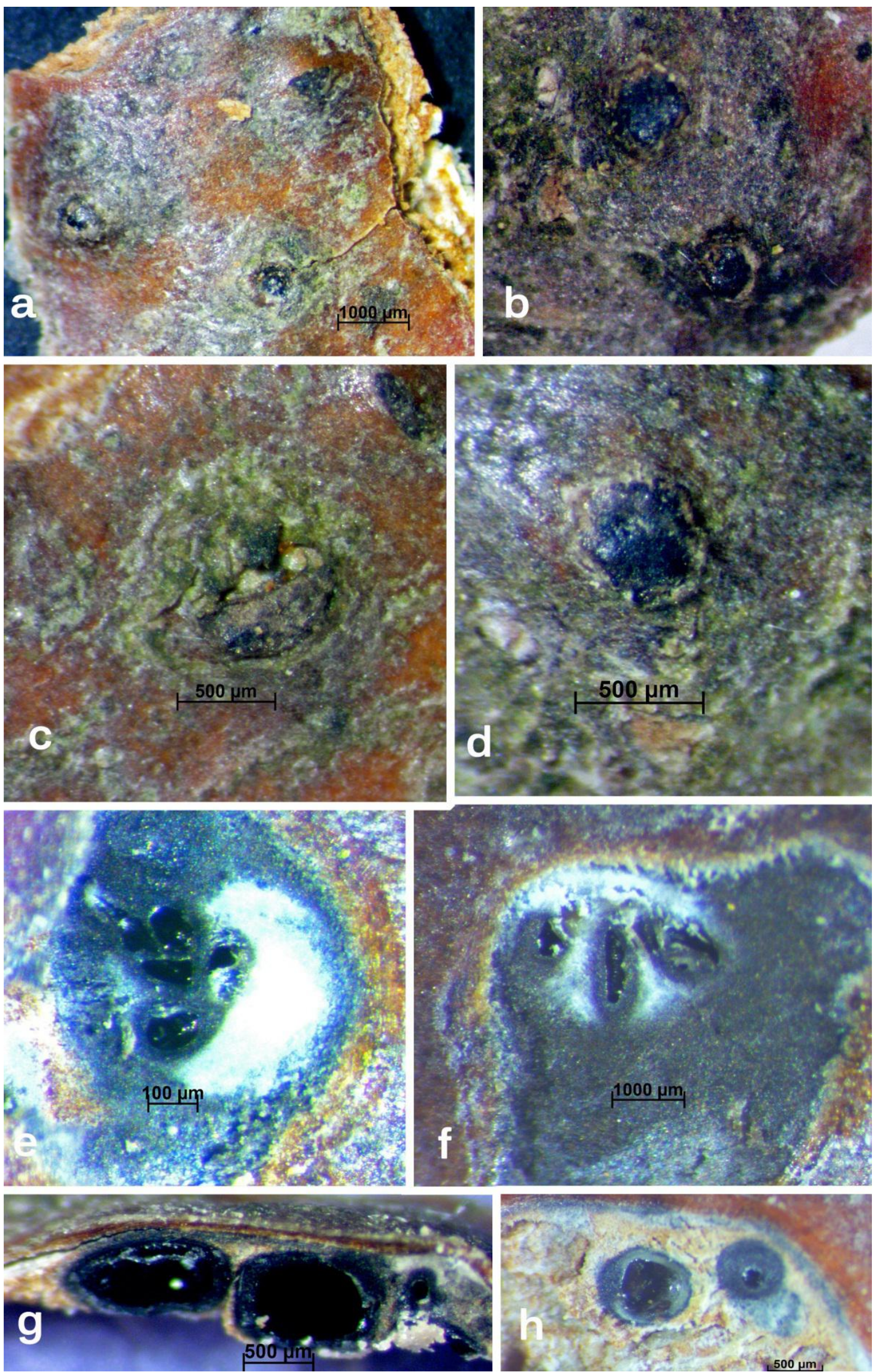

Fig. 3 - Lopadostoma fagi. a-c. Surface of stromata on host, d. ectostromatic disc, e, h. transverse sections of stromata, $f$, g. vertical sections of stromata. Scale bars: $a=1000 \mu \mathrm{m}, \mathrm{b}-\mathrm{d}=500 \mu \mathrm{m}, \mathrm{e}=$ $100 \mu \mathrm{m}, \mathrm{f}=1000 \mu \mathrm{m}, \mathrm{g}, \mathrm{h}=500 \mu \mathrm{m}$ 

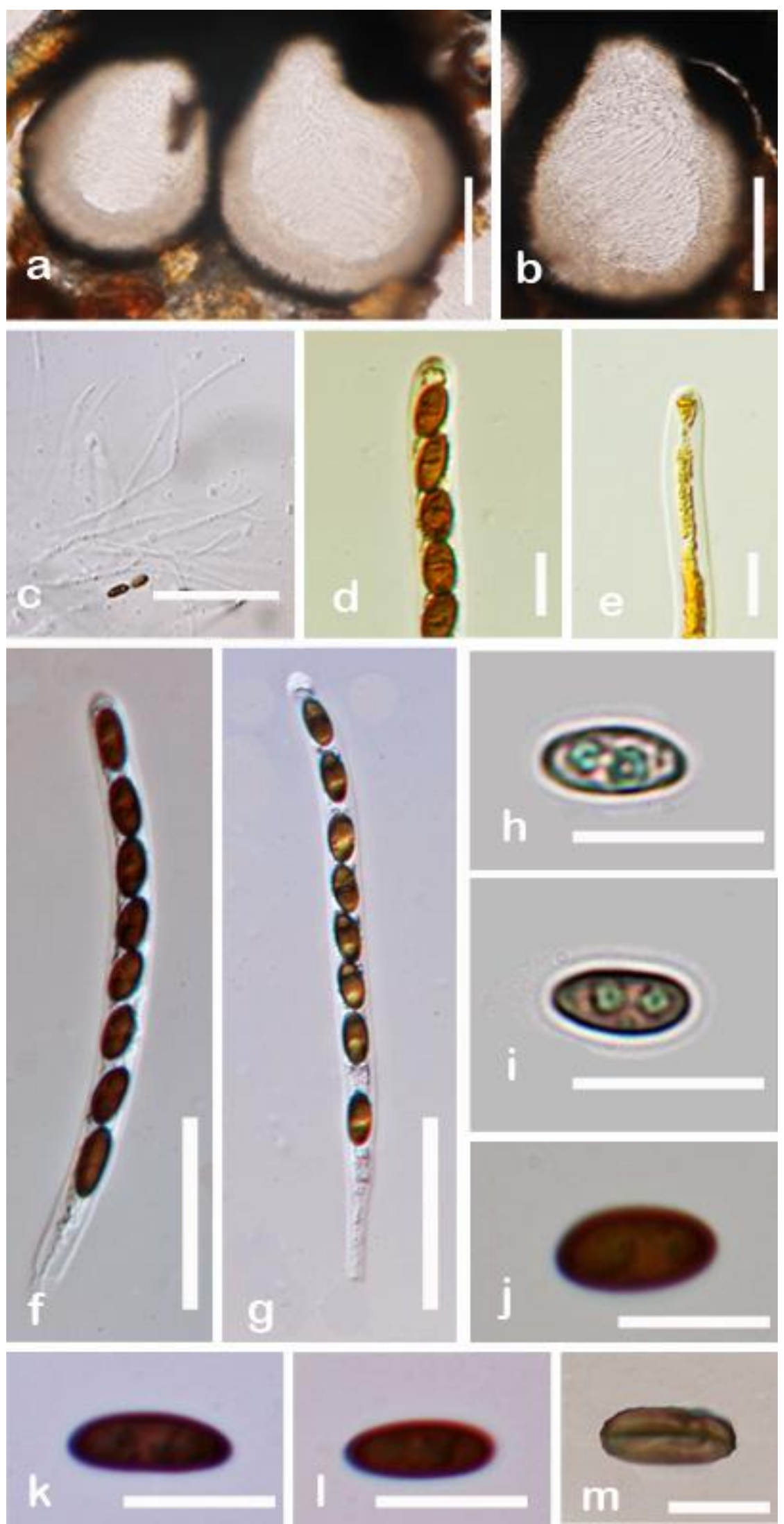

Fig. 4 - Lopadostoma fagi. a, b. Vertical sections of stromata, c. Paraphyses, d, e. Asci with inconspicuous apical apparatus (d-in Melzer's reagent, e-in Lugol solution), f, g. Mature asci, h-i. Immature ascospores, $\mathrm{j}-1$. Mature ascospores, $\mathrm{m}$. Ascospore with straight germ slit. Scale bars: $\mathrm{a}, \mathrm{b}$ $=300 \mu \mathrm{m}, \mathrm{f}=30 \mu \mathrm{m}, \mathrm{d}-\mathrm{e}=10 \mu \mathrm{m}, \mathrm{f}, \mathrm{g}=30 \mu \mathrm{m}, \mathrm{h}-\mathrm{m}=10 \mu \mathrm{m}$. 
and monostichous. In addition there are slight variations in the measurements of perithecia, asci and ascospores which can be due to the geographical variations and environmental conditions. The slow germination of ascospores ( 3 weeks on MEA and 4-5 weeks on OA) later resulted in whitish colonies is similar to those observed by Jaklitsch et al. (2014) who mentioned as 1-4 weeks for initial germination of ascospores on MEA. Due to these morphological similarities between our collection and the previously studied specimens, we have identified this species as L. fagi, which is also confirmed by the molecular studies.

\section{Discussion}

Lopadostoma comprised several unrelated species which were previously introduced by Martin (1969, 1976) until the genus was revised by Rappaz (1995) and recently by Jaklitsch et al. (2014). The latter study limited the genus to 12 species and provided molecular data for ten species. Therefore the genus now comprises only species with pustular stromatic development in the host. Lopadostoma clearly forms a highly supported monophyletic clade in the phylogenetic analysis which is now placed in Lopadostomataceae (Senanayake et al. 2015).

Most of the species in Lopadostoma exhibit overlapping characters which makes it difficult to identify them separately. These variations are caused by changes of climatic conditions during stroma development and maturation as well as based on the host factors (Jaklitsch et al. 2014). Our collection has similar morphological characters of stromata, asci and ascospores with L. fagi. Except those slight variations we observed in asci and ascospores measurements this collection is typical of L. fagi. However, according to Jaklitsch et al. (2014) the qualitative characters are more informative than quantitative characters due to the considerable intraspecific variation.

Therefore molecular data is useful in identification at the species level. Particularly proteincoding genes are superior and useful than other genes such as ITS or LSU (Stadler et al. 2013, Jaklitsch et al. 2014, Daranagama et al. 2015a) since they can provide better resolution at terminal clades and higher statistical supports. The use of RPB2 gene sequences in this study as well as previous studies in combination of ITS and LSU results in better phylogenetic placements, which is also correlated with the morphology and host preference.

The chemical profiles of species of Lopadostoma have not been studied to date. Since the Xylariales are rich in production of secondary metabolites (Stadler and Hellwig 2005, Stadler et al. 2013) the analysis of Lopadostoma species for their secondary metabolites will be useful for a better taxonomic classification.

\section{Acknowledgments}

The authors appreciate the financial support and postgraduate scholarship provided by State Key Laboratory of Mycology, Institute of Microbiology, Chinese Academy of Sciences, Beijing and the Mushroom Research Foundation, Chiang Mai, Thailand.

\section{References}

Daranagama DA, Camporesi E, Tian Q, Liu X, Chamyuang S, Stadler M, Hyde KD. 2015 Anthostomella is polyphyletic comprising several genera in Xylariaceae. Fungal Diversity 73, 203-238 DOI 10.1007/s13225-015-0329-6.

Jaklitsch WM, Fournier J, Rogers JD, Voglmayr H. 2014 - Phylogenetic and taxonomic revision of Lopadostoma. Persoonia 32, 52-82. http://dx.doi.org/10.3767/003158514X679272

Jayasiri SC, Hyde KD, Ariyawansa HA, Bhat J, Buyck B, Cai L, Dai YC, Abd-Elsalam KA, Ertz D, Hidayat I, Jeewon R, Jones EBG, Bahkali AH, Karunarathna SC, Liu JK, Luangsa-ard JJ, Lumbsch HT, Maharachchikumbura SSN, McKenzie EHC, Moncalvo JM, Ghobad-Nejhad M, Nilsson H, Pang KA, Pereira OL, Phillips AJL, Raspé O, Rollins AW, Romero AI, Etayo J, Selçuk F, Stephenson SL, Suetrong S, Taylor JE, Tsui CKM, Vizzini A, AbdelWahab MA, Wen TC, Boonmee S, Dai DQ, Daranagama DA, Dissanayake AJ, Ekanayaka AH, Fryar SC, Hongsanan S, Jayawardena RS, Li WJ, Perera RH, Phookamsak R, de Silva 
NI, Thambugala KM, Tian Q, Wijayawardene NN, Zhao RL, Zhao Q, Kang JC, Promputtha I. 2015 - The Faces of Fungi database: fungal names linked with morphology, phylogeny and human impacts. Fungal Diversty 74, 3-18. doi:10.1007/s13225-015-0351-8.

Ju YM, San Martín F, Rogers JD. 1993 - Three xylariaceous fungi with scolecosporous conidia. Mycotaxon 47, 219-228.

Katoh K, Standley DM. 2013 - MAFFT multiple sequence alignment software version 7: improvements in performance and usability. Molecular Biology and Evolution 30, 772-780.

Læssøe T. 1994 - Index Ascomycetum 1. Xylariaceae. Systema Ascomycetum 13, 43-112.

Lu BS, Hyde KD. 2000 - A world monograph of Anthostomella. Fungal Diversity Research Series $4,1-376$.

Maharachchikumbura SS, Hyde KD, Jones EBG, McKenzie EHC, Huang SK, Abdel-Wahab MA, Daranagama DA, Dayarathne M, D’souza MJ, Goonasekara ID, Hongsanan S, Jayawardena RS, Kirk PM, Konta S, Liu JK, Liu ZY, Norphanphoun C, Pang KL, Perera RH, Senanayake IC, Shang Q, Shenoy BD, Xiao YP, Bahkali AH, Kang JC, Somrothipol S, Suetrong S, Wen TC, Xu JC. 2015 - Towards a natural classification and backbone tree for Sordariomycetes. Fungal Diversity 72, 199-301 DOI 10.1007/s13225-015-0331-z.

Martin PMD. 1969 - Studies in the Xylariaceae: VII. Anthostomella and Lopadostoma. Journal of South African Botany 35: 393-410.

Martin PMD. 1976 - Studies in the Xylariaceae: supplementary note. Journal of South African Botany 42: 71-83.

Miller MA, Pfeiffer W, Schwartz T. 2010 - Creating the CIPRES Science Gateway for inference of large phylogenetic trees. In: Proceedings of the Gateway Computing Environments Workshop (GCE), 14 Nov. 2010, New Orleans, Louisiana.

Nylander JAA. 2004 - MrModeltest v2. Program distributed by the author. Evolutionary biology centre. Uppsala University, Uppsala.

Rambaut A, Drummond A. 2008 - Fig Tree: Tree figure drawing tool, version 1.2. 2. Institute of Evolutionary Biology, University of Edinburgh.

Rappaz F. 1992 - Anthostoma decipiens et sa position systématique. Mycologia Helvetica 5, 21-32.

Rappaz F. 1993 - Germination conidienne des Diatrypacées: rôle du substrat et de l'inoculum mis en évidence chez Anthostoma decipiens. Sydowia 44, 294-306.

Rappaz F. 1995 - Anthostomella and related xylariaceous fungi on hard wood from Europe and North America. Mycologia Helvetica 7, 99-168.

Rogers JD. 1994 - Problem genera and family interfaces in the Eupyrenomycetes. In: Hawksworth, D. L., ed. Ascomycete systematics: Problems and perspectives in the nineties. Plenum Press, New York. p. 321-331.

Senanayake IC, Maharachchikumbura SSN, Hyde KD, Bhat JD, Jones EBG, Mckenzie EHC, Dai DQ, Daranagama DA, Dayarathne MC, Goonasekara ID, Konta S, Li WJ, Shang QJ, Stadler M, Wijayawardene NN, Xiao YP, Norphanphoun C, Li QR, Liu XZ, Bahkali AH, Kang JC, Wang Y, Wen TC, Xu JC, Camporesi E. 2015 - Towards unraveling relationships in Xylariomycetidae (Sordariomycetes). Fungal Diversity 73, 73-144 DOI 10.1007/s13225015-0340-y.

Stadler M, Hellwig V. 2005 - Chemotaxonomy of the Xylariaceae and remarkable bioactive compounds from Xylariales and their associated asexual stages. Recent Research Developments in Phytochemistry 9, 41-93.

Stadler M, Kuhnert E, Peršoh D, Fournier J. 2013 - The Xylariaceae as model example for a unified nomenclature following the "One fungus-one name" (1F1N) concept. Mycology: International Journal on Fungal Biology 4, 5-21. http://dx.doi.org/10.1080/21501203.2013.782478.

Stamatakis A, Hoover P, Rougemont J. 2008 - A rapid bootstrap algorithm for the RAxML web servers. Systematic Biology 57, 758-771.

Stamatakis A. 2006 - RAxML-VI-HPC: maximum likelihood-based phylogenetic analyses with thousands of taxa and mixed models. Bioinformatics 22, 2688-2690. 
Tamura K, Peterson D, Peterson N, Stecher G, Nei M, Kumar S. 2011 - MEGA5: molecular evolutionary genetics analysis using maximum likelihood, evolutionary distance, and maximum parsimony methods. Molecular Biology and Evolution 28, 2731-2739. 